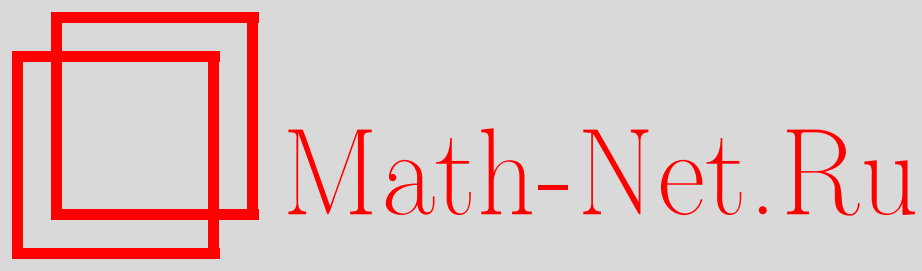

М. Ю. Малышев, Е. В. Прохватилов, Р. А. Зубов, В. А. Франке, Построение пертурбативно корректного гамильтониана на световом фронте для $(2+1)$ мерной калибровочной теории, ТМФ, 2017, том 190, номер 3, 479-493

DOI: https://doi.org/10.4213/tmf9129

Использование Общероссийского математического портала Math-Net.Ru подразумевает, что вы прочитали и согласны с пользовательским соглашением http: //www . mathnet.ru/rus/agreement

Параметры загрузки:

IP : 54.164 .48 .24

26 апреля 2023 г., 14:05:58

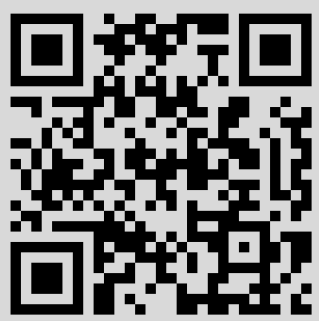




\section{ПОСТРОЕНИЕ ПЕРТУРБАТИВНО КОРРЕКТНОГО ГАМИЛЬТОНИАНА НА СВЕТОВОМ ФРОНТЕ ДЛЯ $(2+1)$-МЕРНОЙ КАЛИБРОВОЧНОЙ ТЕОРИИ}

Обсуждается теория возмущений на световом фронте, регуляризованная методом, аналогичным регуляризации Паули-Вилларса для $(2+1)$-мерной $S U(N)$ симметричной теории калибровочного поля. Это дает возможность построения корректного перенормированного гамильтониана на световом фронте.

Ключевые слова: регуляризация Паули-Вилларса, квантование на световом фронте, теория калибровочных полей.

DOI: https://doi.org/10.4213/tmf9129

\section{1. ВВЕДЕНИЕ}

Настоящая статья является продолжением начатого ранее исследования возможности построения корректного перенормированного гамильтониана на световом фронте (СФ) [1]-[9]. Координаты светового фронта определяются следующим образом [10]:

$$
x^{ \pm}=\frac{x^{0} \pm x^{1}}{\sqrt{2}}, x^{\perp},
$$

где $x^{0}, x^{1}, x^{\perp}$ - лоренцевы координаты. Гамильтониан получается каноническим квантованием на поверхности СФ, т.е. $x^{+}=0$, где $x^{+}$играет роль времени. Для такого квантования характерно появление сингулярности при нулевом значении импульса $p_{-}$. Поэтому необходима регуляризация указанной особенности. Можно ввести эту регуляризацию, ограничивая моды полей условием $\left|p_{-}\right| \geqslant \varepsilon$. Однако

Работа М. Ю. Малышева, Е. В. Прохватилова и Р. А. Зубова выполнена при финансовой поддержке Санкт-Петербургского государственного университета (грант № 11.38.189.2014).

* Петербургский институт ядерной физики им. Б. П. Константинова, Национальный исследовательский центр “Курчатовский институт", Гатчина, Ленинградская обл., Россия

${ }^{\dagger}$ Санкт-Петербургский государственный университет, Санкт-Петербург, Россия. E-mail:m.malyshev@spbu.ru,mimalysh@yandex.ru 
результаты такой теории могут оказаться неэквивалентными результатам, которые получаются при обычном квантовании на поверхности постоянного времени $x^{0}$ в лоренцевых координатах. Так, в рамках теории возмущений по константе связи [1], [11], [12] была обнаружена неэквивалентность между теорией возмущений, генерируемой каноническим гамильтонианом на СФ, и обычной теорией возмущений, соответствующей квантованию на поверхности постоянного времени в лоренцевых координатах. Кроме того, квантование на СФ не позволяет корректно описывать непертурбативные вакуумные эффекты [13], [14].

Отметим также подход к построению эффективного гамильтониана квантовой хромодинамики (КХД) на СФ, предложенный в работах [15]-[17]. Что касается различий между теорией возмущений, генерируемой каноническим гамильтонианом на СФ, и обычной теорией возмущений, соответствующей квантованию на поверхности постоянного времени в лоренцевых координатах, то в работах [1], [2] был найден метод устранения этих отличий путем введения дополнительных полей, которые аналогичны полям, использующимся в регуляризации Паули-Вилларса (ПВ) [18]. В $(3+1)$-мерной КХД [2], рассматриваемой на СФ (в калибровке СФ), введение таких полей нарушает калибровочную симметрию. Была предложена соответствующая ультрафиолетовая (УФ) перенормировка теории, восстанавливающая эту симметрию в пределе снятия регуляризации.

Таким образом, можно построить перенормированный гамильтониан на СФ, пертурбативно эквивалентный гамильтониану теории, квантованной на поверхности постоянного времени в лоренцевых координатах. Однако коэффициенты в перенормировочных контрчленах в таком гамильтониане остаются неизвестными параметрами, поскольку их значения определяются вкладами бесконечного числа диаграмм Фейнмана. Тем не менее, если перейти от $(3+1)$-мерной КХД к ее $(2+1)$-мерному варианту, число таких диаграмм становится конечным (в силу суперперенормируемости этой модели), и можно найти явные выражения для коэффициентов при контрчленах через исходные параметры теории и параметры регуляризации. Такая модель для чисто калибровочных полей была описана в работе [9], но многие результаты, касающиеся анализа диаграмм теории возмущений, были изложены в этой работе достаточно кратко. Кроме того, не было получено выражение для перенормированного гамильтониана на СФ. В настоящей работе мы рассматриваем построение такого гамильтониана, а также приводим некоторые подробности доказательств различных утверждений, имеющихся в работе [9].

\section{2. ВОЗМОЖНЫЕ ОТЛИЧИЯ ТЕОРИИ ВОЗМУЩЕНИЙ НА СФ И НА ПОВЕРХНОСТИ ПОСТОЯННОГО ВРЕМЕНИ В ЛОРЕНЦЕВЫХ КООРДИНАТАХ}

Как и в работе [9], мы рассматриваем модель теории Янга-Миллса в $(2+1)$ мерном пространстве. Диаграммы ковариантной теории возмущений для этой модели могут различаться в теории, квантованной на поверхности постоянного времени в лоренцевых координатах, и в теории, квантованной на СФ, в зависимости от способа регуляризации этих теорий.

В основе сравнения таких диаграмм лежит следующее доказанное в работе [1] утверждение. Обозначим импульсы пропагаторов через $Q_{\mu}=\left(Q_{+}, Q_{-}, Q_{\perp}\right)$, где $Q_{ \pm}=\left(Q_{0} \pm Q_{1}\right) / \sqrt{2}$ - импульсы в координатах С $\Phi$, а $Q_{0}, Q_{1}, Q_{\perp}$ - импульсы 
в лоренцевых координатах. Тогда выражение для диаграммы теории в лоренцевых координатах удается записать в виде следующего интеграла по импульсам в координатах СФ (интегрирование по $Q_{\perp}$ в координатах СФ не отличается от интегрирования в лоренцевых координатах, поэтому данное интегрирование для краткости мы не выписываем) [1]:

$$
\prod_{i} \int d Q_{i+} \int_{B} d Q_{i-} f\left(Q_{i}, p_{j}\right)
$$

где подынтегральное выражение $f\left(Q_{i}, p_{j}\right)$ включает в себя дельта-функции, выражающие законы сохранения импульсов в вершинах, а область интегрирования по $Q_{i-}$ ограничена областью, размер которой имеет порядок наибольшего из внешних импульсов $p_{j-}$ (точнее, ограничена областью размером $s\left|p_{i-}^{\max }\right|$, где константа $s$ зависит от структуры диаграммы).

Если в этом интеграле ввести дополнительное ограничение $\left|Q_{i-}\right| \geqslant \varepsilon$, то получится результат, соответствующий этой диаграмме, вычисленной в теории на СФ, в которой используется обычная регуляризация $\left|Q_{-}\right| \geqslant \varepsilon$. Ограничение интегрирований по $Q_{i-}$ областью $B$ можно связать с аналогичным ограничением в "старой" теории возмущений на СФ, где импульсы $Q_{i-} \geqslant 0$ в промежуточных состояниях не превосходят полного импульса. В результате получаем, что отличие диаграммы в теории на СФ и в лоренцевых координатах определяется только суммой вкладов в интеграл (1) областей, где интегрирование по какому-либо из $Q_{i-}$ ограничено интервалом $\left|Q_{i-}\right| \leqslant \varepsilon$. Далее, как и в работах [1], [7], мы будем называть различные члены этой суммы конфигурациями.

Обратимся к рассматриваемой нами $(2+1)$-мерной теории Янга-Миллса с плотностью лагранжиана

$$
\mathcal{L}=-\frac{1}{4} F_{\mu \nu}^{a} F^{a \mu \nu}+\frac{m}{2} \varepsilon^{\mu \nu \alpha}\left(A_{\mu}^{a} \partial_{\nu} A_{\alpha}^{a}+\frac{2}{3} g f^{a b c} A_{\mu}^{a} A_{\nu}^{b} A_{\alpha}^{c}\right),
$$

где $A_{\mu}^{a}(x)-S U(N)$-симметричное калибровочное поле,

$$
F_{\mu \nu}^{a}=\partial_{\mu} A_{\nu}^{a}-\partial_{\nu} A_{\mu}^{a}+g f_{a b c} A_{\mu}^{b} A_{\nu}^{c},
$$

$a=1, \ldots, N^{2}-1$ суть индексы присоединенного представления, $f_{a b c}$ - структурные константы, $m$ - масса, $g$ - константа связи, $\varepsilon^{\mu \nu \alpha}-$ символ Леви-Чивиты. Здесь добавлен член Черна-Саймонса, необходимый для регуляризации инфракрасных (ИК) расходимостей [19], [20].

Далее мы выбираем калибровку СФ $A_{-}=0$. Это ведет к исчезновению члена четвертой степени по полям $A_{\mu}$.

В предыдущей статье [9] было отмечено, что для УФ-перенормировки теории достаточно перенормировать конечное число диаграмм, перечисленных в указанной статье [9], индекс УФ-расходимости которых не превышает единицы.

Рассмотрим вариант УФ-регуляризации этой теории, в котором мы используем введение как дополнительных полей, так и высших производных. При этом учтем, что выбранная калибровка $A_{-}=0$ порождает дополнительную особенность в пропагаторе глюонного поля (а именно, дополнительный полюс по $p_{-}$). Для регуляризации этой особенности в лагранжиане мы вводим еще одно дополнительное поле и связанный с ним дополнительный параметр регуляризации $\mu$ [9]. 
Начнем с теории с одним дополнительным полем и покажем, что в этой теории имеются отличия между вычислениями диаграмм теории возмущений, полученной при квантовании на поверхности постоянного времени $x^{0}=0$, и теории возмущений, полученной при квантовании на светоподобной поверхности $x^{+}=0$. Регуляризованный таким образом лагранжиан имеет следующий вид:

$$
\begin{aligned}
\mathcal{L}= & -\frac{1}{4} f_{1}^{a \mu \nu}\left(\frac{\Lambda^{2}+2 \partial_{+} \partial_{-}}{\Lambda^{2}}\right) f_{1, \mu \nu}^{a}+\frac{m}{2} \varepsilon^{\mu \nu \alpha} A_{1, \mu}^{a}\left(\frac{\Lambda^{2}+2 \partial_{+} \partial_{-}}{\Lambda^{2}}\right) \partial_{\nu} A_{1, \alpha}^{a}+ \\
& +\frac{1}{4} f_{2}^{a \mu \nu}\left(\frac{\mu^{2}+2 \partial_{+} \partial_{-}}{\mu^{2}}\right) f_{2, \mu \nu}^{a}+\frac{m}{2} \varepsilon^{\mu \nu \alpha} A_{2, \mu}^{a}\left(\frac{\mu^{2}+2 \partial_{+} \partial_{-}}{\mu^{2}}\right) \partial_{\nu} A_{2, \alpha}^{a}+ \\
& +g f^{a b c} A_{\mu}^{a} A_{\nu}^{b} \partial^{\mu} A^{c \nu},
\end{aligned}
$$

где $f_{j, \mu \nu}^{a}=\partial_{\mu} A_{j, \nu}^{a}-\partial_{\nu} A_{j, \mu}^{a}, j=1,2$, величина $A_{1, \mu}^{a}$ - исходное поле, $A_{2, \mu}^{a}$ - дополнительное поле, а в член взаимодействия входит их сумма $A_{\mu}^{a}=A_{1, \mu}^{a}+A_{2, \mu}^{a}$. Кроме того, $A_{j,-}^{a}=0$.

Пропагаторы полей $A_{j \mu}$ записываются как

$$
\begin{aligned}
& \Delta_{1, \mu \nu}^{a b}=\frac{i \delta^{a b}}{Q^{2}-m^{2}+i 0}\left(g_{\mu \nu}-\frac{Q_{\mu} n_{\nu}+n_{\mu} Q_{\nu}+i m \varepsilon_{\mu \nu \alpha} n^{\alpha}}{2 Q_{+} Q_{-}+i 0} 2 Q_{+}\right) \frac{1}{2 Q_{+} Q_{-} / \Lambda^{2}-1+i 0}, \\
& \Delta_{2, \mu \nu}^{a b}=\frac{-i \delta^{a b}}{Q^{2}-m^{2}+i 0}\left(g_{\mu \nu}-\frac{Q_{\mu} n_{\nu}+n_{\mu} Q_{\nu}+i m \varepsilon_{\mu \nu \alpha} n^{\alpha}}{2 Q_{+} Q_{-}+i 0} 2 Q_{+}\right) \frac{1}{2 Q_{+} Q_{-} / \mu^{2}-1+i 0},
\end{aligned}
$$

где $n_{\mu}$ имеет компоненты $n_{+}=1, n_{-}=n_{\perp}=0, n_{\mu} n^{\mu}=0$.

Анализ УФ-расходимостей в теории возмущений с данным лагранжианом показывает, что введенной регуляризации достаточно для устранения этих расходимостей [9]. Поскольку члены взаимодействия зависят только от $A_{\mu}=A_{1, \mu}+A_{2, \mu}$, теорию возмущений можно сформулировать, пользуясь только суммарным пропагатором

$$
\Delta_{\mu \nu}^{a b}=\Delta_{1, \mu \nu}^{a b}+\Delta_{2, \mu \nu}^{a b}=\frac{-i \delta^{a b}}{Q^{2}-m^{2}+i 0}\left(g_{\mu \nu}-\frac{Q_{\mu} n_{\nu}+n_{\mu} Q_{\nu}+i m \varepsilon_{\mu \nu \alpha} n^{\alpha}}{2 Q_{+} Q_{-}+i 0} 2 Q_{+}\right) R,
$$

где

$$
\begin{aligned}
R & =\frac{-1}{2 Q_{+} Q_{-} / \Lambda^{2}-1+i 0}+\frac{1}{2 Q_{+} Q_{-} / \mu^{2}-1+i 0}= \\
& =\frac{2 Q_{+} Q_{-}\left(\mu^{2}-\Lambda^{2}\right)}{\left(2 Q_{+} Q_{-}-\mu^{2}+i 0\right)\left(2 Q_{+} Q_{-}-\Lambda^{2}+i 0\right)} .
\end{aligned}
$$

В пределе $\mu \rightarrow 0, \Lambda \rightarrow \infty$ регуляризация снимается: $R \rightarrow 1$.

Выпишем выражения для пропагатора при различных лоренцевых индексах (для приводимого ниже анализа мы не используем $S U(N)$-индексы, так как в данном случае они несущественны):

$$
\begin{gathered}
\Delta_{++}(Q)=4 Q_{+}^{2} \Delta(Q), \quad \Delta_{+\perp}(Q)=2 Q_{+}\left(Q_{\perp}+i m\right) \Delta(Q), \\
\Delta_{\perp \perp}(Q)=2 Q_{+} Q_{-} \Delta(Q),
\end{gathered}
$$

где

$$
\Delta(Q)=-i\left(\Lambda^{2}-\mu^{2}\right) \prod_{l=0}^{2}\left(2 Q_{+} Q_{-}-m_{l}^{2}+i 0\right)^{-1}
$$

здесь $m_{0}^{2}=m^{2}+Q_{\perp}^{2}, m_{1}=\Lambda, m_{2}=\mu$. 


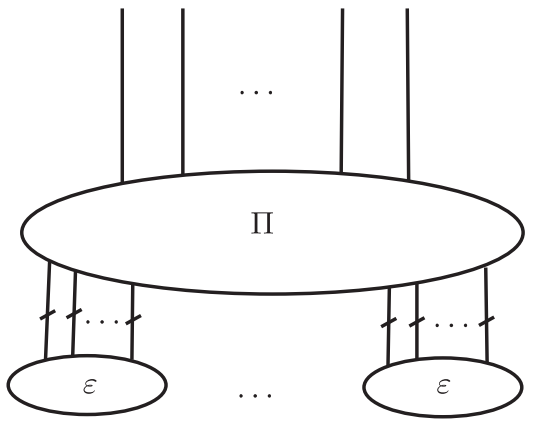

a

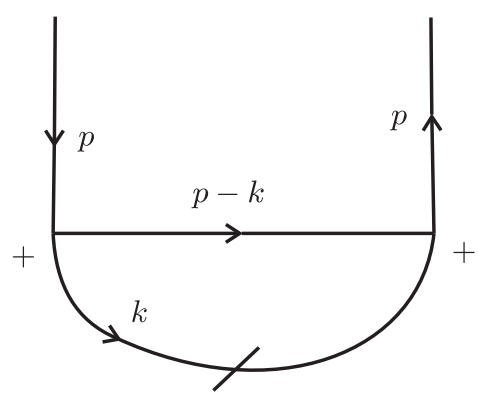

6

Рис. 1. Общий вид конфигурации (а). Простейший пример конфигурации (б).

Напомним, что возможные отличия диаграмм Фейнмана, порожденных квантованием на СФ, от диаграмм Фейнмана в лоренцевых координатах связаны с вкладом в соответствующие интегралы Фейнмана областей интегрирования $\left|Q_{i-}\right| \leqslant \varepsilon$, которые отсутствуют в диаграммах теории на СФ при регуляризации $\left|Q_{i-}\right| \geqslant \varepsilon$. Покажем, что конфигурации, дающие вклад в такое отличие, имеют форму, изображенную на рис. 1а, где перечеркнутые линии обозначают пропагаторы, импульсы которых ограничены условием $\left|Q_{i-}\right| \leqslant \varepsilon$.

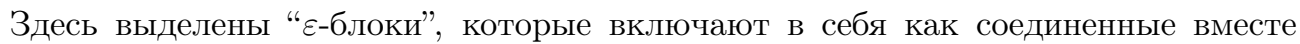
перечеркнутые линии, так и неперечеркнутые линии (называемые далее П-линиями), если их импульсы $Q_{-}$можно ограничить величиной порядка $\varepsilon$ (например, если они присоединены только к перечеркнутым линиям, то в силу утверждения, приведенного в начале данного раздела, эти импульсы ограничены областью $B$ размера порядка $\varepsilon$ ). Кроме того, выделен П-блок, в который входят все остальные П-линии. $\mathrm{K}$ этому блоку подходят все внешние линии. Начнем с простейшего примера такой конфигурации (см. рис. 1б). Соответствующий ей интеграл Фейнмана имеет следующий вид:

$$
\begin{gathered}
I(p)=-4 g^{2} N\left(\Lambda^{2}-\mu^{2}\right)^{2} \int d k_{+} \int_{-\varepsilon}^{\varepsilon} d k_{-} \frac{\left(2 p_{-}-k_{-}\right)^{2}\left(p_{+}-k_{+}\right)\left(p_{-}-k_{-}\right)}{\left(\prod_{l=0}^{2}\left(2\left(p_{+}-k_{+}\right)\left(p_{-}-k_{-}\right)-m_{l}^{2}+i 0\right)\right)} \times \\
\times \frac{k_{+}^{2}}{\prod_{l=0}^{2}\left(2 k_{+} k_{-}-m_{l}^{2}+i 0\right)} .
\end{gathered}
$$

Для оценки этого интеграла в пределе $\varepsilon \rightarrow 0$ устраним зависимость от $\varepsilon$ пределов интегрирования по $k_{-}$, сделав замену $k_{-} \rightarrow k_{-} \varepsilon$, а также проведем дополнительно замену $k_{+} \rightarrow k_{+} / \varepsilon$. После этой замены переменных интегрирования интеграл приобретает вид

$$
\begin{aligned}
& I(p)=-8 g^{2} N\left(\Lambda^{2}-\mu^{2}\right)^{2} \int d k_{+} \int_{-1}^{1} d k_{-} \frac{\left(2 p_{-}-\varepsilon k_{-}\right)^{2}\left(p_{+}-k_{+} / \varepsilon\right)\left(p_{-}-\varepsilon k_{-}\right)}{\prod_{l=0}^{2}\left(2\left(p_{+}-k_{+} / \varepsilon\right)\left(p_{-}-\varepsilon k_{-}\right)-m_{l}^{2}+i 0\right)} \times \\
& \times \frac{k_{+}^{2} / \varepsilon^{2}}{\prod_{l=0}^{2}\left(2 k_{+} k_{-}-m_{l}^{2}+i 0\right)}=
\end{aligned}
$$




$$
\begin{aligned}
=-8 g^{2} N\left(\Lambda^{2}-\mu^{2}\right)^{2} \int d k_{+} \int_{-1}^{1} d k_{-} & \frac{\left(2 p_{-}-\varepsilon k_{-}\right)^{2}\left(\varepsilon p_{+}-k_{+}\right)\left(p_{-}-\varepsilon k_{-}\right)}{\prod_{l=0}^{2}\left(2\left(\varepsilon p_{+}-k_{+}\right)\left(p_{-}-\varepsilon k_{-}\right)-\varepsilon m_{l}^{2}+i 0\right)} \times \\
& \times \frac{k_{+}^{2}}{\prod_{l=0}^{2}\left(2 k_{+} k_{-}-m_{l}^{2}+i 0\right)}
\end{aligned}
$$

Поскольку интеграл по $k_{+}$сходится, в пределе $\varepsilon \rightarrow 0$ получается следующее конечное выражение:

$$
-4 g^{2} N\left(\Lambda^{2}-\mu^{2}\right)^{2} \int d k_{+} \int_{-1}^{1} d k_{-} \frac{1}{\prod_{l=0}^{2}\left(2 k_{+} k_{-}-m_{l}^{2}+i 0\right)},
$$

т. е. имеется отличие между расчетом рассматриваемой диаграммы при квантовании на СФ и обычным ковариантным расчетом в лоренцевых координатах.

Чтобы провести оценку зависимости от $\varepsilon$ в общем виде, рассмотрим сначала, как меняются пропагаторы при вышеуказанной замене переменных интегрирования. Пусть пропагаторный импульс $\left|Q_{-}\right| \leqslant \varepsilon$. Как и на рис. $1 б$, будем вместо $Q$ писать $k$ $\left(\right.$ так что $\left|k_{-}\right| \leqslant \varepsilon$ ). Совершим замены $k_{+} \rightarrow k_{+}^{\prime}=\varepsilon k_{+}, k_{-} \rightarrow k_{-}^{\prime}=k_{-} / \varepsilon$. Тогда согласно формуле (6) для компонент пропагатора получаем

$$
\begin{aligned}
\Delta(k) \rightarrow \Delta^{\prime}\left(k^{\prime}\right)=\Delta\left(k^{\prime}\right) \sim O(1), & \Delta_{++}(k) \rightarrow \Delta_{++}^{\prime}\left(k^{\prime}\right)=\frac{\Delta_{++}\left(k^{\prime}\right)}{\varepsilon^{2}}, \\
\Delta_{+\perp}(k) \rightarrow \Delta_{+\perp}^{\prime}\left(k^{\prime}\right)=\frac{\Delta_{+\perp}\left(k^{\prime}\right)}{\varepsilon}, & \Delta_{\perp \perp}(k) \rightarrow \Delta_{\perp \perp}^{\prime}\left(k^{\prime}\right)=\Delta_{\perp \perp}\left(k^{\prime}\right) \sim O(1) .
\end{aligned}
$$

Пусть пропагаторный импульс $Q_{\mu}=p_{\mu}-k_{\mu}$, где импульс $p_{-}$конечен, а $\left|k_{-}\right| \leqslant \varepsilon$. Тогда пропагатор меняется следующим образом:

$$
\begin{aligned}
\Delta(p-k) & \rightarrow \Delta^{\prime}\left(p, k^{\prime}\right)= \\
& =\frac{-i\left(m_{1}^{2}-m_{2}^{2}\right) \varepsilon^{3}}{\left(-p_{-}+\varepsilon k_{-}^{\prime}\right)^{3} \prod_{l=0}^{2}\left(2 k_{+}^{\prime}-2 \varepsilon p_{+}+\varepsilon m_{l}^{2} /\left(p_{-}-\varepsilon k_{-}^{\prime}\right)+i 0 / p_{-}\right)} \sim O\left(\varepsilon^{3}\right), \\
\Delta_{++}(p-k) & \rightarrow \frac{4\left(\varepsilon p_{+}-k_{+}^{\prime}\right)^{2} \Delta^{\prime}\left(p, k^{\prime}\right)}{\varepsilon^{2}} \sim O(\varepsilon), \\
\Delta_{+\perp}(p-k) & \rightarrow \frac{2\left(\varepsilon p_{+}-k_{+}^{\prime}\right)\left(p_{\perp}-k_{\perp}+i m\right) \Delta^{\prime}\left(p, k^{\prime}\right)}{\varepsilon} \sim O\left(\varepsilon^{2}\right), \\
\Delta_{\perp \perp}(p-k) & \rightarrow \frac{2\left(\varepsilon p_{+}-k_{+}^{\prime}\right)\left(p_{-}-\varepsilon k_{-}^{\prime}\right) \Delta^{\prime}\left(p, k^{\prime}\right)}{\varepsilon} \sim O\left(\varepsilon^{2}\right) .
\end{aligned}
$$

Все вершинные множители можно оценить конечной величиной, кроме тех случаев, когда в вершине сходятся три $\varepsilon$-линии (тогда они оцениваются величиной порядка $\varepsilon)$. Используя эти оценки, легко доказать, что при любом другом выборе индексов в диаграмме на рис. 16 она стремится к нулю при $\varepsilon \rightarrow 0$. Добавление к $\varepsilon$-линии дополнительных $\varepsilon$-линий, т. е. создание $\varepsilon$-блока, не изменяет оценки по $\varepsilon$ диаграммы на рис. 1б. Это можно показать следующим образом: $\varepsilon$-блок состоит из вершин и пропагаторов, каждая вершина пропорциональна $\varepsilon$ и имеет один индекс + , который отвечает тому, что в присоединенном к этой вершине пропагаторе появляется множитель $k_{+}^{\prime} / \varepsilon$. Это ведет к сокращению зависимости от $\varepsilon$ в данной вершине. Это справедливо как для пропагаторов, имеющих индексы ++ и дающих вклад, пропорциональный $1 / \varepsilon^{2}$, так и для пропагаторов, имеющих индексы $+\perp$ 


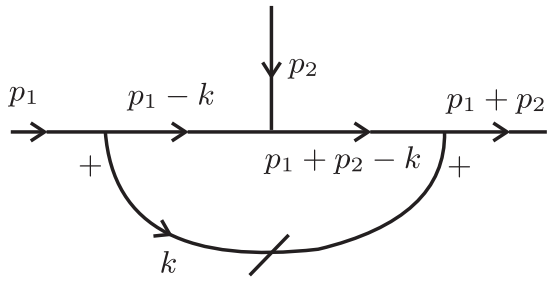

a

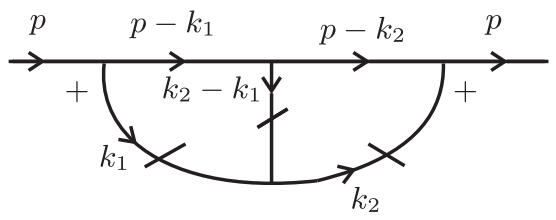

б

Рис. 2. Усложнения простейшей конфигурации.

и дающих вклад, пропорциональный $1 / \varepsilon$. Пропагатор с индексами $\perp \perp$ дает вклад

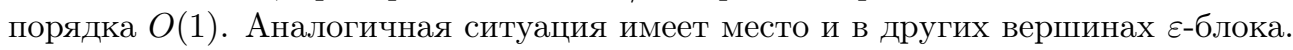

Следующее простое изменение конфигурации на рис. 16 имеет вид, изображенный на рис. 2a, который отличается от рис. 16 добавлением одной внешней линии. В такой диаграмме присутствуют две П-линии, через которые проходит импульс $k$ $\varepsilon$-линий. Поэтому, используя оценки, соответствующие формулам (10) и (11), получаем, что конфигурация стремится к нулю при $\varepsilon \rightarrow 0$. Аналогичная ситуация получается при дальнейшем увеличении числа П-линий, через которые проходит импульс $k$-линий, например, из-за увеличения числа внешних линий.

Рассмотрим еще один случай изменения конфигурации на рис. 1б, когда добавляется одна $\varepsilon$-линия, соединяющая П- и $\varepsilon$-линии исходной конфигурации (см. рис. 2б). В этом случае П- и $\varepsilon$-линии исходной конфигурации разделяются на две части, где импульсы $k_{1}$ и $k_{2}$ пропагаторов разделенной $\varepsilon$-линии можно выбрать в качестве петлевых переменных интегрирования так, что они будут проходить через один из пропагаторов разделенной П-линии (см. рис. 2б). Тогда оценки, соответствующие формулам (10) и (11), и учет вклада новой вершины, присоединенной к исходной $\varepsilon$ линии, приведут к тому, что конфигурация стремится к нулю при $\varepsilon \rightarrow 0$.

Таким образом, наиболее общий вид ненулевой конфигурации может быть изображен по аналогии с диаграммой на рис. 1б, в которой перечеркнутая линия заменяется на произвольный $\varepsilon$-блок.

В итоге мы приходим к заключению, что в данной теории имеются ненулевые конфигурации, причем все они конечны в пределе $\varepsilon \rightarrow 0$.

Теперь покажем, что предложенный в статье [9] метод добавления еще одного вспомогательного поля позволяет сделать нулевыми рассмотренные выше ненулевые конфигурации. В этом случае лагранжиан имеет вид

$$
\begin{aligned}
\mathcal{L}=\sum_{l=1}^{3}( & \left.-\frac{r_{l}}{4} f_{l}^{a \mu \nu}\left(\frac{m_{l}^{2}+2 \partial_{+} \partial_{-}}{M_{l}^{2}}\right) f_{l, \mu \nu}^{a}+r_{l} \frac{m}{2} \varepsilon^{\mu \nu \alpha} A_{l, \mu}^{a}\left(\frac{m_{l}^{2}+2 \partial_{+} \partial_{-}}{M_{l}^{2}}\right) \partial_{\nu} A_{l, \alpha}^{a}\right)+ \\
& +g f^{a b c} A_{\mu}^{a} A_{\nu}^{b} \partial^{\mu} A^{c \nu}
\end{aligned}
$$

где $A_{1, \mu}^{a}$ - исходное поле, а $A_{2, \mu}^{a}$ и $A_{3, \mu}^{a}$ - дополнительные поля, $A_{l,-}^{a}=0, A_{\mu}^{a}=$ $A_{1, \mu}^{a}+A_{2, \mu}^{a}+A_{3, \mu}^{a}$, параметры $m, m_{1}=\Lambda, m_{2}=\mu$ аналогичны параметрам в лагранжиане (4), $r_{l}= \pm 1$. В выражении (12) $m_{3}$ - новый параметр, связанный с введением дополнительного поля $A_{3, \mu}^{a}$. Величины $M_{l}^{2}$ выражаются через параметры $m_{l}$ и $m$ так, чтобы индекс УФ-расходимости суммарного пропагатора уменьшился еще на 
две единицы. Суммарный пропагатор есть сумма пропагаторов каждого поля:

$\Delta_{\mu \nu}^{a b}=\frac{-i \delta^{a b}}{\left(k^{2}-m^{2}+i 0\right)}\left(g_{\mu \nu}-\frac{k_{\mu} n_{\nu}+n_{\mu} k_{\nu}+i m \varepsilon_{\mu \nu \alpha} n^{\alpha}}{2 k_{+} k_{-}+i 0} 2 k_{+}\right)\left(\sum_{l=1}^{3} \frac{r_{l} M_{l}^{2}}{m_{l}^{2}-2 k_{+} k_{-}-i 0}\right)$.

Если привести сумму, входящую в формулу (13), к общему знаменателю и потребовать исчезновения членов четвертой и нулевой степени по $k$ в числителе этой суммы, то получим следующие условия:

$$
\sum_{l=1}^{3} r_{l} M_{l}^{2}=0, \quad \sum_{l=1}^{3} \frac{r_{l} M_{l}^{2}}{m_{l}^{2}}=0
$$

Далее потребуем, чтобы полученное выражение для суммарного пропагатора переходило в выражение для пропагатора нерегуляризованной теории при $m_{2}^{2}=\mu^{2} \rightarrow 0$, $m_{1}^{2}=\Lambda^{2} \rightarrow \infty, m_{3}^{2} \rightarrow \infty$, т. е.

$$
\begin{aligned}
2 k_{+} k_{-}- & \frac{r_{1} M_{1}^{2}\left(m_{2}^{2}+m_{3}^{2}\right)+r_{2} M_{2}^{2}\left(m_{1}^{2}+m_{3}^{2}\right)+r_{3} M_{3}^{2}\left(m_{1}^{2}+m_{2}^{2}\right)}{\prod_{l=1}^{3}\left(2 k_{+} k_{-}-m_{l}^{2}+i 0\right)} \rightarrow \\
& \rightarrow \frac{r_{1} M_{1}^{2} m_{3}^{2}+r_{2} M_{2}^{2}\left(m_{1}^{2}+m_{3}^{2}\right)+r_{3} M_{3}^{2} m_{1}^{2}}{m_{1}^{2} m_{3}^{2}}= \\
& =r_{1} \frac{M_{1}^{2}}{m_{1}^{2}}+r_{2}\left(\frac{1}{m_{1}^{2}}+\frac{1}{m_{3}^{2}}\right) M_{2}^{2}+r_{3} \frac{M_{3}^{2}}{m_{3}^{2}} \rightarrow 1
\end{aligned}
$$

Если учесть второе равенство в (14), то условие (15) можно удовлетворить, полагая, например, $r_{2} M_{2}^{2} / m_{2}^{2}=-1$. При этом согласно соотношениям (14) получаем

$$
r_{1} M_{1}^{2}=\frac{m_{1}^{2}\left(m_{3}^{2}-m_{2}^{2}\right)}{m_{3}^{2}-m_{1}^{2}}, \quad r_{3} M_{3}^{2}=-\frac{m_{3}^{2}\left(m_{1}^{2}-m_{2}^{2}\right)}{m_{3}^{2}-m_{1}^{2}} .
$$

Кроме того, потребуем, чтобы в пределе снятия регуляризации $m_{2}^{2} \rightarrow 0, m_{1}^{2} \rightarrow \infty$, $m_{3}^{2} \rightarrow \infty$ оставалось только поле $A_{1}$. Для этого наложим дополнительное условие $m_{3}^{2} / m_{1}^{2} \rightarrow \infty$. Из этих фактов видно, что $r_{1}=1, r_{2}=-1, r_{3}=-1$.

Чтобы оценить зависимость различных конфигураций от $\varepsilon$, перепишем суммарный пропагатор в следующем виде:

$$
\Delta_{\mu \nu}^{a b}(Q)=\frac{i \delta^{a b}\left(2 Q_{+} Q_{-} g_{\mu \nu}-2 Q_{+}\left(Q_{\mu} n_{\nu}+Q_{\nu} n_{\mu}+i m \varepsilon_{\mu \nu \alpha} n^{\alpha}\right)\right)}{\prod_{l=0}^{3}\left(2 Q_{+} Q_{-}-m_{l}^{2}+i 0\right)} R,
$$

где

$$
m_{0}^{2}=m^{2}+Q_{\perp}^{2}, \quad R=r_{1} M_{1}^{2}\left(m_{2}^{2}+m_{3}^{2}\right)+r_{2} M_{2}^{2}\left(m_{1}^{2}+m_{3}^{2}\right)+r_{3} M_{3}^{2}\left(m_{1}^{2}+m_{2}^{2}\right) .
$$

При этом отвечающие соотношениям (10) оценки для пропагатора (17) остаются прежними, а оценки, отвечающие соотношениям (11), принимают следующий вид:

$$
\begin{aligned}
\Delta^{\prime}\left(p, k^{\prime}\right) & \sim O\left(\varepsilon^{4}\right), & \Delta_{++}^{\prime}\left(p, k^{\prime}\right) & \sim O\left(\varepsilon^{2}\right), \\
\Delta_{+\perp}^{\prime}\left(p, k^{\prime}\right) & \sim O\left(\varepsilon^{3}\right), & \Delta_{\perp \perp}^{\prime}\left(p, k^{\prime}\right) & \sim O\left(\varepsilon^{3}\right),
\end{aligned}
$$




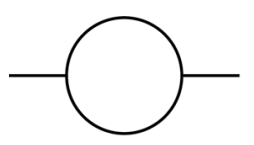

a

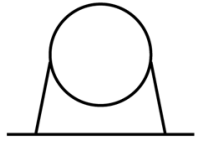

б

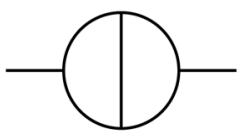

B

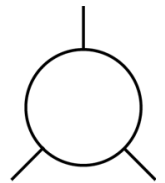

Рис. 3. Диаграммы, расходящиеся в пределе снятия УФ-регуляризации.

т. е. степень величины $\varepsilon$ увеличивается по сравнению с формулами (11). Это приводит к исчезновению в пределе $\varepsilon \rightarrow 0$ всех рассмотренных выше ненулевых конфигураций. Таким образом, в теории с лагранжианом (12) мы получаем совпадение значений диаграмм при квантовании на поверхности постоянного времени в лоренцевых координатах и при квантовании на СФ.

С использованием результатов работы [2] в работе [9] было показано, что ИК-расходимостей, связанных с параметром регуляризации $\mu$, удается избежать в пределе снятия регуляризации, если выполнено условие $(\ln \mu)^{n} / \Lambda \rightarrow 0$ при $\mu \rightarrow 0, \Lambda \rightarrow \infty$, где $n$ - произвольное целое число (например, можно положить $\mu=m^{2} / \Lambda$ ).

Поскольку рассматриваемая теория суперперенормируема в $(2+1)$-мерном пространстве, требуется проанализировать и вычислить конечное число УФ-расходящихся диаграмм, изображенных на рис. 3.

В работе [9] было показано, что однопетлевая диаграмма с двумя хвостами на рис. За, имеющая индекс УФ-расходимости, равный единице, и однопетлевая диаграмма с тремя хвостами и с индексом УФ-расходимости, равным нулю, изображенная на рис. Зг, оказываются конечными при точном аналитическом вычислении. Оставшиеся две двухпетлевые диаграммы с двумя хвостами (см. рис. 3б, Зв), имеющие индекс УФ-расходимости, равный нулю, могут быть перенормированы путем сравнения этих диаграмм с соответствующими перенормированными диаграммами при их размерной регуляризации. При этом перенормировочные контрчлены определяются с помощью вычисления разности выражений для этих диаграмм в рассматриваемой регуляризации и в размерной регуляризации. Такая процедура перенормировки позволяет восстановить калибровочную и лоренцеву симметрии в теории с лагранжианом (12) в пределе снятия регуляризации.

В результате к лагранжиану (12) добавляется контрчлен вида $C \ln (\Lambda / m) A_{\perp}^{a} A_{\perp}^{a}$, где константу $C$ можно определить с помощью численного анализа диаграмм, изображенных на рис. 3б, Зв. Такая форма контрчлена согласуется с остаточной лоренц-инвариантностью лагранжиана (12) относительно преобразований координат $x^{+} \rightarrow \lambda x^{+}, x^{-} \rightarrow \lambda^{-1} x^{-}, x^{\perp} \rightarrow x^{\perp}$. Вследствие этой инвариантности логарифмически расходящиеся диаграммы на рис. 3б, 3в, вычисляемые при нулевом внешнем импульсе (и при внешних лоренцевых индексах, отличных от индекса -), обращаются в ноль, если содержат хотя бы один внешний лоренцев индекс + . Таким образом, вклад в контрчлен дают диаграммы, соответствующие только внешним лоренцевым индексам $\perp$. В приложении мы приводим выражение для диаграммы, изображенной на рис. 3 a. 


\section{3. ПОСТРОЕНИЕ ПЕРЕНОРМИРОВАННОГО ГАМИЛЬТОНИАНА НА СФ}

Рассмотрим теорию с лагранжианом (12). Напишем выражение для этого лагранжиана, полагая $A_{l,-}^{a}=0$ :

$$
\begin{aligned}
\mathcal{L}=\sum_{l=1}^{3} r_{l}( & -\frac{\partial_{+} f_{l,+-}^{a} \partial_{-} f_{l,+-}^{a}}{M_{l}^{2}}+\frac{m_{l}^{2}}{2 M_{l}^{2}}\left(f_{l,+-}^{a}\right)^{2}+ \\
& +\partial_{+} A_{l, \perp}^{a} \frac{2 \partial_{-}^{2}}{M_{l}^{2}} \partial_{+} A_{l, \perp}^{a}+\frac{m_{l}^{2}}{M_{l}^{2}} \partial_{+} A_{l, \perp}^{a} \partial_{-} A_{l, \perp}^{a}+ \\
& +m\left(\frac{m_{l}^{2}}{M_{l}^{2}} f_{l,+-}^{a} A_{l, \perp}^{a}+\frac{2}{M_{l}^{2}} f_{l,+-}^{a} \partial_{+} \partial_{-} A_{l, \perp}^{a}\right)- \\
& \left.-\partial_{\perp} f_{l,+-}^{a}\left(\frac{m_{l}^{2}+2 \partial_{+} \partial_{-}}{M_{l}^{2}}\right) A_{l, \perp}^{a}\right)-g f^{a b c} A_{+}^{a} A_{\perp}^{b} \partial_{-} A_{\perp}^{c} .
\end{aligned}
$$

Чтобы привести действие, соответствующее этому лагранжиану, к гамильтоновой форме, найдем сначала импульс $\prod_{l, \perp}^{a}$, сопряженный к полю $A_{l, \perp}^{a}$ :

$$
\Pi_{l, \perp}^{a}=\frac{\partial \mathcal{L}}{\partial\left(\partial_{+} A_{l, \perp}^{a}\right)}=r_{l}\left(4 \frac{\partial_{-}^{2}}{M_{l}^{2}} \partial_{+} A_{l, \perp}^{a}+\frac{m_{l}^{2}}{M_{l}^{2}} \partial_{-} A_{l, \perp}^{a}+\frac{2}{M_{l}^{2}}\left(\partial_{\perp}-m\right) \partial_{-} f_{l,+-}^{a}\right) .
$$

Отсюда можно выразить величины $\partial_{+} A_{l, \perp}^{a}$ через переменные $\Pi_{l, \perp}^{a}, A_{l, \perp}^{a}$ и $f_{l,+-}^{a}$ :

$$
\partial_{+} A_{l, \perp}^{a}=\frac{M_{l}^{2}}{4} \partial_{-}^{-2}\left(r_{l} \Pi_{l, \perp}^{a}-\frac{m_{l}^{2}}{M_{l}^{2}} \partial_{-} A_{l, \perp}^{a}-\frac{2}{M_{l}^{2}}\left(\partial_{\perp}-m\right) \partial_{-} f_{l,+-}^{a}\right) .
$$

В результате действие можно переписать как

$$
S=\int d x^{+} d x^{-} d x^{\perp}\left[\sum_{l=1}^{3}\left(-\frac{r_{l}}{M_{l}^{2}} \partial_{+} f_{l,+-}^{a} \partial_{-} f_{l,+-}^{a}+\Pi_{l, \perp}^{a} \partial_{+} A_{l, \perp}^{a}\right)-\mathcal{H}\right],
$$

где

$$
\begin{aligned}
\mathcal{H}\left(\Pi_{l, \perp}^{a},\right. & \left.A_{l, \perp}^{a}, f_{l,+-}^{a}\right)=\sum_{l=1}^{3}\left[-\frac{r_{l} m_{l}^{2}}{2 M_{l}^{2}} f_{l,+-}^{a} f_{l,+-}^{a}+\frac{r_{l} m_{l}^{2}}{M_{l}^{2}} A_{l, \perp}^{a}\left(\partial_{\perp}-m\right) f_{l,+-}^{a}+\right. \\
& +\frac{r_{l} M_{l}^{2}}{8}\left(r_{l} \Pi_{l, \perp}^{a}-\frac{m_{l}^{2}}{M_{l}^{2}} \partial_{-} A_{l, \perp}^{a}-\frac{2}{M_{l}^{2}}\left(\partial_{\perp}-m\right) \partial_{-} f_{l,+-}^{a}\right) \times \\
& \left.\times \partial_{-}^{-2}\left(r_{l} \Pi_{l, \perp}^{a}-\frac{m_{l}^{2}}{M_{l}^{2}} \partial_{-} A_{l, \perp}^{a}-\frac{2}{M_{l}^{2}}\left(\partial_{\perp}-m\right) \partial_{-} f_{l,+-}^{a}\right)\right]+ \\
& +g f^{a b c} A_{+}^{a} A_{\perp}^{b} \partial_{-} A_{\perp}^{c}-C \ln \left(\frac{\Lambda}{m}\right) A_{\perp}^{a} A_{\perp}^{a}
\end{aligned}
$$

Из выражения (22) видно, что канонический импульс, сопряженный к полю $f_{l,+-}^{a}$, порождает на СФ каноническую связь, которая оказывается связью второго рода. 
Чтобы избежать этой связи, перейдем к новым переменным с помощью преобразования Фурье по координате $x^{-}$(вводя регуляризацию $\left|p_{-}\right| \geqslant \varepsilon$ ):

$$
\begin{aligned}
f_{l,+-}^{a}(x) & =\frac{M_{l}}{\sqrt{2 \pi}} \int_{\mid p_{-}>\varepsilon} \frac{d p_{-}}{\sqrt{2\left|p_{-}\right|}} a_{l}^{a}\left(p_{-} ; x^{\perp}, x^{+}\right) e^{-i p_{-} x^{-}}= \\
& =\frac{M_{l}}{\sqrt{2 \pi}} \int_{\varepsilon}^{\infty} \frac{d p_{-}}{\sqrt{2 p_{-}}}\left(a_{l}^{a}\left(p_{-} ; x^{\perp}, x^{+}\right) e^{-i p_{-} x^{-}}+a_{l}^{a+}\left(p_{-} ; x^{\perp}, x^{+}\right) e^{i p_{-} x^{-}}\right) .
\end{aligned}
$$

Тогда член в действии

$$
\sum_{l=1}^{3} \int d x^{+} d x^{-} d x^{\perp}\left(-\frac{r_{l}}{M_{l}^{2}} \partial_{+} f_{l,+-}^{a} \partial_{-} f_{l,+-}^{a}\right)
$$

преобразуется (после интегрирования по частям по $x^{+}$и отбрасывания внеинтегральных членов) к следующему виду:

$$
\sum_{l=1}^{3} \int d x^{+} d x^{\perp} \int_{\varepsilon}^{\infty} d p_{-}\left(-i r_{l} a_{l}^{a+}\left(p_{-} ; x^{\perp}, x^{+}\right) \partial_{+} a_{l}^{a}\left(p_{-} ; x^{\perp}, x^{+}\right)\right) .
$$

Полученная гамильтонова форма действия позволяет получить квантовые перестановочные соотношения для канонических переменных:

$$
\begin{gathered}
{\left[a_{l}^{a}\left(p_{-} ; x^{\perp}, x^{+}\right), a_{l^{\prime}}^{b+}\left(p_{-}^{\prime} ; x^{\prime \perp}, x^{+}\right)\right]=-r_{l} \delta_{l l^{\prime}} \delta^{a b} \delta\left(p_{-}-p_{-}^{\prime}\right) \delta\left(x^{\perp}-x^{\perp}\right),} \\
{\left[A_{l, \perp}^{a}(x), \Pi_{l^{\prime}, \perp}^{b}\left(x^{\prime}\right)\right]_{x^{\prime+}=x^{+}}=i \delta_{l l^{\prime}} \delta^{a b} \delta\left(x^{-}-x^{\prime-}\right) \delta\left(x^{\perp}-x^{\perp}\right) .}
\end{gathered}
$$

Остальные канонические перестановочные соотношения для этих полей равны нулю. Переменные $a_{l}^{a}\left(p_{-} ; x^{\perp}, x^{+}\right)$и $a_{l}^{a+}\left(p_{-} ; x^{\perp}, x^{+}\right)$можно рассматривать как операторы, аналогичные операторам рождения и уничтожения на СФ. Введем также преобразование Фурье для переменных $A_{l, \perp}^{a}(x)$ и $\Pi_{l, \perp}^{b}(x)$ :

$$
\begin{gathered}
A_{l, \perp}^{a}(x)=\frac{M_{l}}{m_{l} \sqrt{2 \pi}} \int_{\mid p_{-\mid>\varepsilon}} \frac{d p_{-}}{\sqrt{2\left|p_{-}\right|}}\left(\left(a_{l, \perp}^{a}\left(p_{-} ; x^{\perp}, x^{+}\right)+a_{l, \perp}^{a+}\left(-p_{-} ; x^{\perp}, x^{+}\right)\right) e^{-i p_{-} x^{-}}\right)= \\
=\frac{M_{l}}{m_{l}} \int_{\varepsilon}^{\infty} \frac{d p_{-}}{\sqrt{4 \pi p_{-}}}\left[\left(a_{l, \perp}^{a}\left(p_{-} ; x^{\perp}, x^{+}\right)+b_{l, \perp}^{a}\left(p_{-} ; x^{\perp}, x^{+}\right)\right) e^{-i p_{-} x^{-}}+\right. \\
\left.+\left(a_{l, \perp}^{a+}\left(p_{-} ; x^{\perp}, x^{+}\right)+b_{l, \perp}^{a+}\left(p_{-} ; x^{\perp}, x^{+}\right)\right) e^{i p_{-} x^{-}}\right]
\end{gathered}
$$

$$
\begin{aligned}
\Pi_{l, \perp}^{b}(x) & =\frac{-i m_{l}}{M_{l} \sqrt{2 \pi}} \int_{\left|p_{-}\right|>\varepsilon} d p_{-} \sqrt{\frac{\left|p_{-}\right|}{2}}\left(\left(a_{l, \perp}^{b}\left(p_{-} ; x^{\perp}, x^{+}\right)-a_{l, \perp}^{b+}\left(-p_{-} ; x^{\perp}, x^{+}\right)\right) e^{-i p_{-} x^{-}}\right)= \\
& =\frac{-i m_{l}}{M_{l}} \int_{\varepsilon}^{\infty} d p_{-} \sqrt{\frac{p_{-}}{4 \pi}}\left[\left(a_{l, \perp}^{b}\left(p_{-} ; x^{\perp}, x^{+}\right)-b_{l, \perp}^{b}\left(p_{-} ; x^{\perp}, x^{+}\right)\right) e^{-i p_{-} x^{-}}-\text {э.c. }\right],
\end{aligned}
$$

где введено обозначение $b_{l, \perp}^{a}\left(p_{-} ; x^{\perp}, x^{+}\right)=a_{l, \perp}^{a+}\left(-p_{-} ; x^{\perp}, x^{+}\right)$.

Операторы $a_{l, \perp}^{a}\left(p_{-} ; x^{\perp}, x^{+}\right), a_{l, \perp}^{a+}\left(p_{-} ; x^{\perp}, x^{+}\right), b_{l, \perp}^{a}\left(p_{-} ; x^{\perp}, x^{+}\right), b_{l, \perp}^{a+}\left(p_{-} ; x^{\perp}, x^{+}\right)$удовлетворяют следующим каноническим перестановочным соотношениям, аналогичным перестановочным соотношениям для операторов рождения и уничтожения:

$$
\begin{aligned}
{\left[a_{l, \perp}^{a}\left(p_{-} ; x^{\perp}, x^{+}\right), a_{l^{\prime}, \perp}^{b+}\left(p_{-}^{\prime} ; x^{\perp}, x^{+}\right)\right] } & =\delta_{l l^{\prime}} \delta^{a b} \delta\left(p_{-}-p_{-}^{\prime}\right) \delta\left(x^{\perp}-x^{\perp}\right), \\
{\left[b_{l, \perp}^{a}\left(p_{-} ; x^{\perp}, x^{+}\right), b_{l^{\prime}, \perp}^{b+}\left(p_{-}^{\prime} ; x^{\prime \perp}, x^{+}\right)\right] } & =-\delta_{l l^{\prime}} \delta^{a b} \delta\left(p_{-}-p_{-}^{\prime}\right) \delta\left(x^{\perp}-x^{\perp}\right) ;
\end{aligned}
$$


остальные коммутаторы равны нулю. В терминах этих операторов свободная (квадратичная по полям) часть гамильтониана принимает следующий вид:

$$
\begin{aligned}
& H=\int d x^{\perp} \int_{\varepsilon}^{\infty} d p_{-} \sum_{l=1}^{3} \frac{r_{l}}{2 p_{-}}\left[-m_{l}^{2} a_{l}^{a+}\left(p_{-} ; x^{\perp}, x^{+}\right) a_{l}^{a}\left(p_{-} ; x^{\perp}, x^{+}\right)-\right. \\
& -\left(\partial_{\perp}-m\right) a_{l}^{a+}\left(p_{-} ; x^{\perp}, x^{+}\right)\left(\partial_{\perp}-m\right) a_{l}^{a}\left(p_{-} ; x^{\perp}, x^{+}\right)+ \\
& +m_{l}\left(\left(\partial_{\perp}-m\right) a_{l}^{a+}\left(p_{-} ; x^{\perp}, x^{+}\right)\left(a_{l, \perp}^{a}\left(p_{-} ; x^{\perp}, x^{+}\right)+b_{l, \perp}^{a}\left(p_{-} ; x^{\perp}, x^{+}\right)\right)+\text {э.c. }\right)- \\
& -m_{l}^{2}\left(\frac{1-r_{l}}{2} a_{l, \perp}^{a+}\left(p_{-} ; x^{\perp}, x^{+}\right)+\frac{1+r_{l}}{2} b_{l, \perp}^{a+}\left(p_{-} ; x^{\perp}, x^{+}\right)\right) \times \\
& \quad \times\left(\frac{1-r_{l}}{2} a_{l, \perp}^{a}\left(p_{-} ; x^{\perp}, x^{+}\right)+\frac{1+r_{l}}{2} b_{l, \perp}^{a}\left(p_{-} ; x^{\perp}, x^{+}\right)\right)+ \\
& +m_{l}\left(\left(\partial_{\perp}-m\right) a_{l}^{a+}\left(p_{-} ; x^{\perp}, x^{+}\right) \times\right. \\
& \left.\left.\quad \times\left(\frac{1-r_{l}}{2} a_{l, \perp}^{a}\left(p_{-} ; x^{\perp}, x^{+}\right)+\frac{1+r_{l}}{2} b_{l, \perp}^{a}\left(p_{-} ; x^{\perp}, x^{+}\right)\right)+\text {э.c. }\right)\right] .
\end{aligned}
$$

Введение новых переменных, аналогичных операторам рождения и уничтожения, дает в гамильтониане достаточно простую зависимость от $m_{l}$. Однако квадратичная по полям часть гамильтониана все еще не имеет диагонального вида. В принципе, ее можно привести к диагональному виду, но получающиеся при этом выражения имеют достаточно громоздкий вид.

\section{4. ЗАКЛЮЧЕНИЕ}

В представленной работе рассмотрено построение перенормированного гамильтониана на СФ для $S U(N)$-калибровочно-инвариантной теории Янга-Миллса в случае $(2+1)$-мерного пространства-времени. В этом случае теория возмущений по константе взаимодействия суперперенормируема и требуется перенормировать только конечное число УФ-расходящихся диаграмм. Это позволяет найти в явном виде контрчлены, перенормирующие теорию, используя только исходные параметры теории. С другой стороны, для восстановления эквивалентности во всех порядках теории возмущений по константе связи между получаемой теорией на СФ и обычной теорией, квантованной на поверхности постоянного времени в лоренцевых координатах, потребовалось ввести дополнительные поля, аналогичные полям, которые используются при регуляризации Паули-Вилларса. Эти поля порождают пространство Фока на СФ с индефинитной метрикой. Проведение непертурбативных расчетов с таким гамильтонианом на СФ является достаточно трудной задачей [8], [21]-[24].

В работе [25] был рассмотрен простейший пример: расчет спектра ангармонического осциллятора, модифицированного введением дополнительных степеней свободы, аналогичных дополнительным полям регуляризации Паули-Вилларса. В этом случае достаточно хорошо видно, как часть спектра, связанная с дополнительными степенями свободы, отделяется от обычного спектра модели и стремится к бесконечным значениям в пределе, соответствующем снятию регуляризации Паули-Вилларса.

В качестве альтернативного подхода к построению перенормированного гамильтониана на СФ можно указать подход, описанный в работах [16], [17] и в работе [15] 
(в которой также приведены все необходимые ссылки на предыдущие работы). Этот подход использует метод частичной диагонализации гамильтониана с помощью унитарного преобразования, зависящего от масштабного параметра. Данное преобразование строится с использованием теории возмущений по константе взаимодействия. Таким образом, появляется возможность записать приближенное выражение для перенормированного гамильтониана в терминах эффективных операторов рождения и уничтожения "составных частиц". Однако указанная процедура достаточно громоздка и ограничивается только низшими порядками теории возмущений.

\section{Вычисление диаграмм, определяющих контрчлен в перенормированном гамильтониане}

ПРИЛОЖЕНИЕ

Согласно формуле (17) пропагатор обращается в ноль, если хотя бы один его лоренцев индекс положить равным -, т. е. $\Delta_{\mu-}^{a b}=0$. Из пропагатора при остальных лоренцевых индексах можно сформировать следующую матрицу:

$$
\Delta^{a b}(k)=\left(\begin{array}{cc}
\Delta_{++}^{a b}(k) & \Delta_{+\perp}^{a b}(k) \\
\Delta_{\perp+}^{a b}(k) & \Delta_{\perp \perp}^{a b}(k)
\end{array}\right)=\frac{i \delta^{a b}}{k^{2}-m^{2}+i 0}\left(\begin{array}{cc}
2 k_{+} & k_{\perp}-i m \\
k_{\perp}+i m & k_{-}
\end{array}\right) .
$$

Вершине соответствует следующий множитель:

$$
V^{\mu \nu \rho, a b c}(p, k, q)=-g f^{a b c}\left((p-k)^{\rho} g^{\mu \nu}+(k-q)^{\mu} g^{\nu \rho}+(q-p)^{\nu} g^{\mu \rho}\right) .
$$

$\mathrm{У}$ метрического тензора $g^{\mu \nu}$ отличны от нуля только компоненты $g^{+-}=g^{-+}=1$, $g^{\perp \perp}=-1$. Поскольку в диаграммах для функций Грина к вершинам подсоединяются пропагаторы, нет необходимости рассматривать вершины с лоренцевыми индексами -; заметим, что это потребовалось, если бы мы использовали явно тождества Уорда. Фиксируя один лоренцев индекс, можно сформировать следующую матрицу из вершины при остальных лоренцевых индексах:

$$
\begin{aligned}
V^{\mu, a b c}(p, k, q) & =\left(\begin{array}{cc}
V^{\mu++, a b c}(p, k, q) & V^{\mu+\perp, a b c}(p, k, q) \\
V^{\mu \perp+, a b c}(p, k, q) & V^{\mu \perp \perp, a b c}(p, k, q)
\end{array}\right)= \\
& =-g f^{a b c}\left(\begin{array}{cc}
0 & (q-p)_{-} g^{\mu \perp} \\
(p-k)_{-} g^{\mu \perp} & (k-q)_{\perp} g^{\mu \perp}+(q-k)^{\mu}
\end{array}\right) .
\end{aligned}
$$

Для диаграммы, изображенной на рис. За, получается следующее выражение:

$$
\begin{aligned}
& D^{\mu_{1} \mu_{2}, a_{1} a_{2}}(k)= \\
& =\int d q_{+} d q_{-} d q_{\perp}\left(V^{\mu_{1} \nu_{1} \rho_{1}, a_{1} b_{1} c_{1}}(k, q) \Delta_{\rho_{1} \nu_{2}}^{c_{1} b_{2}}(k+q) V^{\mu_{2} \nu_{2} \rho_{2}, a_{2} b_{2} c_{2}}(k, q) \Delta_{\rho_{2} \nu_{1}}^{c_{2} b_{1}}(q)\right)= \\
& =-g^{2} N \delta^{a_{1} a_{2}} \int \frac{d q_{+} d q_{-} d q_{\perp}}{\left(q^{2}-m^{2}+i 0\right)\left((k+q)^{2}-m^{2}+i 0\right)} \times \\
& \quad \times \operatorname{Tr}\left\{\left(\begin{array}{cc}
0 & \left(q_{-}+2 k_{-}\right) g^{\mu_{1} \perp} \\
\left(q_{-}-k_{-}\right) g^{\mu_{1} \perp} & 2 q^{\mu_{1}}+k^{\mu_{1}}-\left(k_{\perp}+2 q_{\perp}\right) g^{\mu_{1} \perp}
\end{array}\right) \times\right. \\
& \quad \times\left(\begin{array}{cc}
2\left(k_{+}+q_{+}\right) & k_{\perp}+q_{\perp}-i m \\
k_{\perp}+q_{\perp}+i m & k_{-}-q_{-}
\end{array}\right) \times \\
& \left.\quad \times\left(\begin{array}{cc}
0 & \left(q_{-}-k_{-}\right) g^{\mu_{2} \perp} \\
\left(q_{-}+2 k_{-}\right) g^{\mu_{2} \perp} & 2 q^{\mu_{2}}+k^{\mu_{2}}-\left(k_{\perp}+2 q_{\perp}\right) g^{\mu_{2} \perp}
\end{array}\right)\left(\begin{array}{cc}
2 q_{+} & q_{\perp}-i m \\
q_{\perp}+i m & q_{-}
\end{array}\right)\right\} .
\end{aligned}
$$


Здесь была использована формула $f^{a_{1} b_{1} b_{2}} f^{a_{2} b_{1} b_{2}}=N \delta^{a_{1} a_{2}}$. Чтобы вычислить контрчлен, мы рассматриваем разность выражений для этого интеграла в нашей регуляризации и в размерной регуляризации, учитывая первые два члена ряда Тейлора по $k$ в окрестности точки $k=0$. Кроме того, мы совершаем евклидов поворот в импульсном пространстве. В результате легко убедиться, что все компоненты диаграммы, содержащие лоренцев индекс + , исчезают. Оставшаяся компонента с индексами $\perp \perp$ может быть вычислена аналитически и дает конечный ответ в пределе снятия УФ-регуляризации.

Аналогичное выражение можно написать для диаграммы, изображенной на рис. Зг. Поскольку эта диаграмма может расходиться только логарифмически, мы вычисляем ее при нулевых внешних импульсах. Выражение для этой диаграммы, несмотря на громоздкий вид, допускает аналитическое рассмотрение после евклидова поворота в импульсном пространстве. В результате видно, что при нулевых внешних импульсах эта диаграмма равна нулю. Выражения для оставшихся логарифмически расходящихся диаграмм с интересующими нас лоренцевыми индексами $\perp \perp$, изображенных на рис. 3б, 3в, можно получить аналогичным образом, используя формулы (П.1), (П.3). Но в данном случае эти выражения оказываются намного более громоздкими. Кроме того, их не удается рассмотреть аналитически. Возможны только приближенные численные расчеты, результаты которых тем не менее можно использовать в непертурбативном гамильтоновом подходе к данной модели.

Благодарности. Авторы благодарят С. А. Пастона и М. В. Компанийца за полезные обсуждения, а также организаторов V конференции "Модели квантовой теории поля" (MQFT-2015), посвященной 75-летию со дня рождения А. Н. Васильева.

\section{Список литературы}

[1] С. А. Пастон, В.А. Франке, "Сравнение квантово-полевой теории возмущений на световом фронте и в лоренцевых координатах", ТМФ, 112:3 (1997), 399-416, arXiv: hep-th/9901110.

[2] С. А. Пастон, Е. В. Прохватилов, В.А. Франке, "К построению гамильтониана КХД в координатах светового фронта", ТМФ, 120:3 (1999), 417-437, arXiv: hep-th/0002062.

[3] С. А. Пастон, Е. В. Прохватилов, В. А. Франке, "Гамильтонов формализм на световом фронте для двумерной квантовой электродинамики, эквивалентный лоренц-ковариантному подходу", ТМФ, 131:1 (2002), 84-97, arXiv: hep-th/0302016.

[4] S. A. Paston, E. V. Prokhvatilov, V.A. Franke, "On the correspondence between a light-front hamiltonian approach and a Lorentz-covariant formulation for quantum gauge theories", Nucl. Phys. B Proc. Suppl., 108:1-3 (2002), 189-193, arXiv: hep-th/0111009.

[5] S. J. Brodsky, V. A. Franke, J. R. Hiller, G. McCartor, S. A. Paston, E. V. Prokhvatilov, "A nonperturbative calculation of the electron's magnetic moment", Nucl. Phys. B, 703:1-2 (2004), 333-362, arXiv: hep-ph/0406325.

[6] С. А. Пастон, Е. В. Прохватилов, В. А. Франке, "Вычисление спектра масс КЭД-2 в координатах светового фронта", ЯФ, 68:2 (2005), 292-303, arXiv: hep-th/0501186.

[7] V.A. Franke, Yu. V. Novozhilov, S. A. Paston, E. V. Prokhvatilov, "Quantization of field theory on the light front", Focus on Quantum Field Theory, ed. O. Kovras, Nova Sci. Publ., New York, 2005, 23-81, arXiv: hep-th/0404031.

[8] M. Yu. Malyshev, S. A. Paston, E. V. Prokhvatilov, R. A. Zubov, "Renormalized light front Hamiltonian in the Pauli-Villars regularization", Internat. J. Theor. Phys., 54:1 (2015), 169-184, arXiv: 1311.4381. 
[9] М. Ю. Малышев, С. А. Пастон, Е. В. Прохватилов, Р. А. Зубов, В. А. Франке, "Регуляризация Паули-Вилларса и гамильтониан на световом фронте в $(2+1)$-мерной теории Янга-Миллса", ТМФ, 184:3 (2015), 487-498, arXiv: 1505.00272.

[10] P. A. M. Dirac, "Forms of relativistic dynamics", Rev. Modern Phys., 21:3 (1949), 392-399.

[11] M. Burkardt, A. Langnau, "Hamiltonian formulation of $(2+1)$-dimensional QED on the light cone", Phys. Rev. D, 44:4 (1991), 1187-1197.

[12] M. Burkardt, A. Langnau, "Rotational invariance in light-cone quantization", Phys. Rev. D, 44:12 (1991), 3857-3867.

[13] Е. В. Прохватилов, В. А. Франке, “Предельный переход к светоподобным координатам в теории поля и КХД-гамильтониан", ЯФ, 49:4 (1989), 1109-1117.

[14] Е.-М. Ильгенфриц, С.А. Пастон, Г.-Ю. Пирнер, Е. В. Прохватилов, В. А. Франке, "Квантовые поля на световом фронте, формулировка в координатах, близких к световому фронту, решеточное приближение", ТМФ, 148:1 (2006), 89-101, arXiv: hep-th/0610020.

[15] S. D. Głazek, M. Gómez-Rocha, "Asymptotic freedom of gluons in the Fock space", Acta Phys. Pol. B, 47:1 (2016), 225-242, arXiv: 1510.01609.

[16] S. D. Głazek, K. G. Wilson, "Perturbative renormalization group for Hamiltonians", Phys. Rev. D, 49:8 (1993), 4214-4218.

[17] S. D. Głazek, K. G. Wilson, "Renormalization of Hamiltonians", Phys. Rev. D, 48:12 (1993), 5863-5872.

[18] W. Pauli, F. Villars, "On the invariant regularization in relativistic quantum theory", Rev. Modern Phys., 21:3 (1949), 434-444.

[19] S. Deser, R. Jackiw, S. Templeton, "Topologically massive gauge theories", Ann. Phys., 281:1-2 (2000), 409-449.

[20] S. Deser, R. Jackiw, S. Templeton, "Three-dimensional massive gauge theories", Phys. Rev. Lett., 48:15 (1982), 975-978.

[21] S. J. Brodsky, J. R. Hiller, G. McCartor, "Application of Pauli-Villars regularization and discretized light-cone quantization to a single-fermion truncation of Yukawa theory", Phys. Rev. D, 64:11 (2001), 114023, 13 pp., arXiv: hep-ph/0107038.

[22] S. J. Brodsky, J. R. Hiller, G. McCartor, "Exact Solutions to Pauli-Villars-regulated field theories", Ann. Phys., 296:2 (2002), 406-424, arXiv: hep-th/0107246.

[23] S. J. Brodsky, J. R. Hiller, G. McCartor, "Application of Pauli-Villars regularization and discretized light-cone quantization to a $(3+1)$-dimensional model", Phys. Rev. D, 60:5 (1999), 054506, 9 pp., arXiv: hep-ph/9903388.

[24] S. J. Brodsky, J. R. Hiller, G. McCartor, "Pauli-Villars regulator as a nonperturbative ultraviolet regularization scheme in discretized light-cone quantization", Phys. Rev. D, 58:2 (1998), 025005, 16 pp., arXiv: hep-th/9802120.

[25] M. Yu. Malyshev, S.A. Paston, E. V. Prokhvatilov, R. A. Zubov, V. A. Franke, "PauliVillars regularization in nonperturbative Hamiltonian approach on the light front", AIP Conf. Proc., 1701:1 (2016), 100012, 9 pp., arXiv: 1504.07951. 